\title{
Change management to initiate and accelerate gender equality
}

\author{
Jemimah Bailey and Eileen Drew
}

\section{Introduction}

One of the central purposes of the Horizon 2020 Systemic Action for Gender Equality (SAGE) project was the implementation of Gender Equality Plans (GEPs). This was in recognition that substantial structural change in academia is needed before gender equality can be achieved. GEPs were developed and implemented as part of this process of structural change in each SAGE implementing institution namely: Instituto Universitário De Lisboa (ISCTE-IUL), Portugal; International University of Sarajevo (IUS), Bosnia and Herzegovina; Kadir Has University, Istanbul (KHAS), Turkey; Sciences Po Bordeaux (SciPo), France; Università degli Studi di Brescia (UNIBS), Italy, under the guidance of the SAGE Coordinating partner Trinity College, Dublin (TCD). In order to facilitate the adoption and integration of the GEPs, a SAGE Model for Institutional Change was designed. This model was informed by the work of organisational change experts such as Kotter $(2012)$ and Kanter $(1977,1989)$ and the experiences, learning and outputs of the FP7 2011-2015 INstitutional Transformation for Effecting Gender Equality in Research (INTEGER) project.

This chapter starts by presenting some of the key themes found in change management literature, particularly those pertaining to gender equality in universities. It then presents the SAGE Model for Institutional Change detailing the nine key components to support the management of change in the creation of a gendersensitive institution. The chapter draws upon SAGE project evaluation reports and an online focus group conducted with SAGE project partners to collect their descriptions of, and reflections on, the change process in promoting gender equality in their institutions.

\section{Change management and gender equality}

The achievement of gender equality can be regarded as both a challenge and a mechanism for change in research-performing organisations, implying systemic, integrated, long-term approaches rather than piecemeal short-term measures. There is a growing body of research that outlines the specific challenges, particularly in a European context, driven by the European Union's commitment to 
gender mainstreaming, adopted in the 1990s as the principal strategy for increasing gender equality:

Gender mainstreaming is a structural transformation strategy that aims to transform organisational processes and practices by eliminating gender biases from existing routines.

(Benschop and Verloo 2011, 283)

Danowitz presents some of the key organisational issues that need attention if gender equality is to be achieved in academia, pointing out that 'changing universities to achieve gender equality ultimately means changing organisational structures and cultures and, at times, the larger policy spheres in which they function' $(2008,96)$. She argues that there are five key factors that must be tackled in the advancement of equality, to ensure that gender equality measures are part of deep cultural change within universities:

- gender equality measures must be grounded in the university's basic values and strategic action plan;

- various constituencies within the university must buy in or accept the proposed gender equality measures;

- gender equality initiatives must be tailored to the particular needs of the university and must be adapted to its mission and culture;

- gender equality initiatives must be linked to major programmes and endeavours;

- a gender equality monitoring system with accountability must be put in place to assess short-range and long-range outcomes (Danowitz 2008, 97).

Established literature on institutional and organisational change within the academia has generally focused on the challenges of adapting to an increasingly globalised and neoliberal world and meeting the demands of expanded access to third-level education. As O'Connor et al note: 'neoliberalism values competition, metrics and financial profit, over collective interests, cooperation and community' $(2019,723)$. One aspect of neoliberalism which has had particular impact in the higher education sector is managerialism, resulting in the adoption of managerial practices drawn from the corporate world. The entrepreneurial university (Clark 1998, 2004) and Sporn's model of university adaptation (1999) elaborate on how higher education institutions change their structures in response to environmental forces. Sporn's review of the current environment for higher education notes that universities:

are still in the process of finding the right balance between demand and response. This is further triggered by stark societal developments like digitalisation, immigration and integration and inequality. 
She suggests that for successful organisational transformation, it is necessary for higher education institutions to use the theories and approaches (including strategic planning, leadership, vision and mission, accountability and impact) offered by organisational studies to steer change efficiently and effectively.

This chapter draws upon organisational change management literature to explain the components deemed relevant to promoting gender equality, demanding new policies, behaviours and actions to meet the many challenges that resist such change in the university sector. Kanter (1989) saw the combination of five major building blocks (Figure 10.1) present in organisational changes, to increase the capacity to meet new challenges. These are described in some detail later in the chapter, with examples of contemporary relevance in academia.
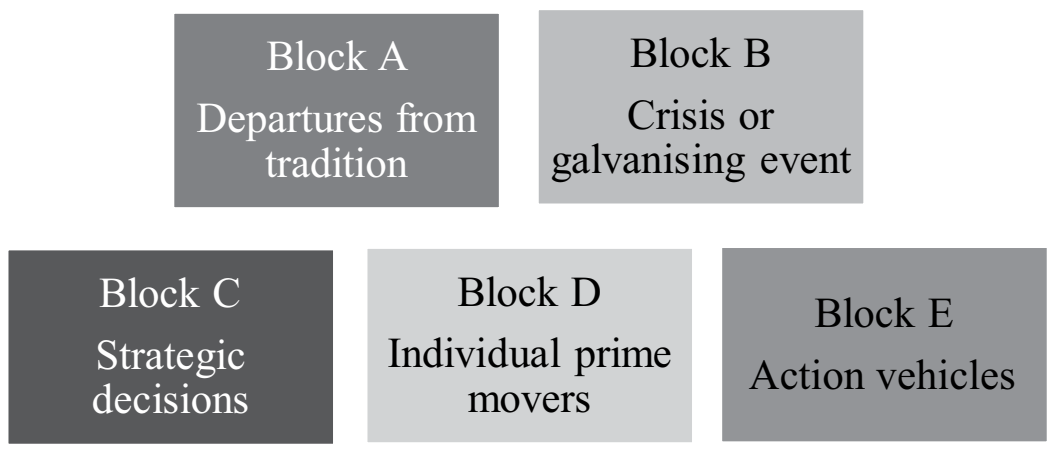

Figure 10.1 Building blocks for change

Source: Moss Kanter (1983)

\section{Block A: Departures from tradition}

These can be orchestrated by innovators, or just happen to the organisation in a passive way. Such departures can provide the university with an incentive to solve new problems or replace existing methods with more productive ones. For these departures to arise, the institution should be loosely enough controlled to promote innovative interventions, in this case a Gender Equality Plan (GEP). However, in themselves, even successful departures (including GEPs) do not produce major change in the absence of other building blocks.

\section{Block B: Crisis or galvanising event}

This refers to external forces such as interventions from higher education funders, or political changes such as Brexit and internal forces provoked by national or sectoral policy changes, or the attitudes of employees. These can be used deliberately to create a change climate. For example, large-scale Japanese organisations 
have been known to identify, or even create, crises in order to galvanise marketing strategies.

\section{Block C: Strategic decisions}

Strong leaders of change are needed to articulate direction by creating a vision, allowing themselves and others to see more clearly the steps to take, building on present capacities and strengths to get there. This can often involve a series of smaller decisions. The process is assisted by more integrative systems, innovations, communication channels and team mechanisms to keep gender equality ideas circulating. These forms of coalitions and cooperative traditions make it easier to get moving. Strategic decisions supporting gender equality help to set in motion the next two major blocks in effecting change.

\section{Block D: Individual 'prime movers'}

No strategy, no matter how brilliant or responsive, can succeed without someone giving it a push — hence the role of change agents who remain committed to the vision, keeping up the momentum and pushing beyond the actions of the innovating team. Prime movers, or champions, raise the new concept or practice on every possible occasion, in every speech, at every meeting, contributing to a shared vision of gender equality. The message is clear and unequivocal, indicating firm commitment. Where change emanates from outside, for example, via the EU or government regulation, it must be internalised in a way that demonstrates how the necessary change will meet organisational needs, for example with targeted Gender Equality Plans, now required in all Irish higher education institutions (HEA 2016). This requirement provides a signal about the direction of change and the need to adapt the organisation's culture in response to the national funding body (Chapter 12).

\section{Block E: Action vehicles}

These are the mechanisms that allow the new action possibilities to be articulated as actual policies and procedures, structures such as teams, communication channels, appraisal measures, work recognition or rewards that should be incorporated into a Gender Equality Plan. Collectively, these building blocks pave the way for significant institutional change which is further examined in terms of Kotter's eight-step change model (2012), which provides a useful source of change management theory and practice. Kotter's model was used to drive structural change for gender equality during the FP7 INTEGER project (2011-2015) and led to the development of the approach adopted by the SAGE project. Kotter's influence on the INTEGER (2015) project is outlined here (Figure 10.2). 


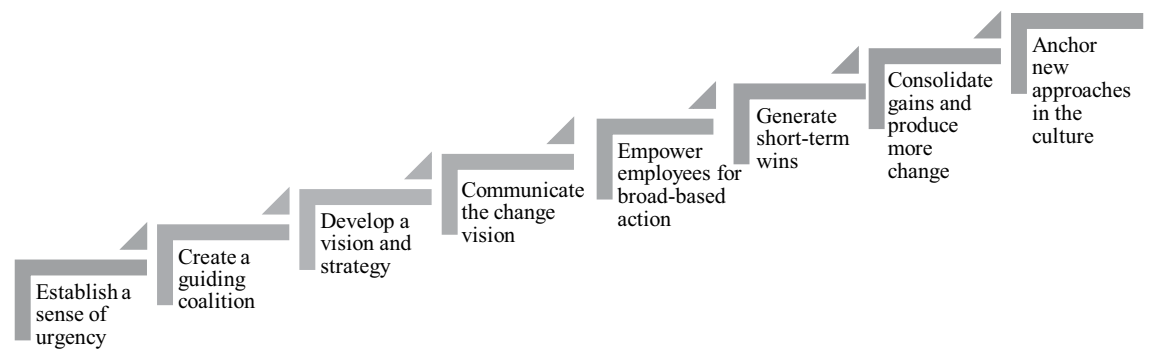

Figure 10.2 Eight-step change model

Source: Kotter 2012

Step 1: Establish a sense of urgency to spark the initial motivation. Like Kanter (1983), Kotter (2012) recommended identifying potential threats or opportunities, developing future scenarios, generating discussions and providing people with convincing reasons for supporting change, aligned with support from external stakeholders. He stressed that this requires high-level buy-in along with time and energy.

Step 2: Create a guiding coalition through strong leadership and visible support from key people (stakeholders and leaders) in the organisation's hierarchy, bringing them together as a change coalition, working as a team to build urgency and momentum for change, appealing for their emotional commitment.

Step 3: Develop a vision and strategy that people can understand and remember through determining the values that are central to change and providing a short summary of what the vision should be.

Step 4: Communicate the change vision by sending a powerful and frequent message to all involved, addressing people's concerns and anxieties openly and honestly, applying the vision to all operations and leading by example.

Step 5: Empower employees for broad-based action by identifying any processes or structures that are barriers to change, thereby assisting them towards executing the vision to move change forward. This can be achieved through new appointments, examining the structures, job descriptions and performance and compensation systems, recognising and rewarding people engaged in change, identifying resistors, helping them and generally removing barriers, human or otherwise.

Step 6: Generate short-term wins early in the change process to help convince critics and negative thinkers, via smaller, inexpensive and achievable targets, having reviewed the pros and cons of each change initiative. Kotter also advocated rewarding the people who help in achieving these targets.

Step 7: Consolidate gains and produce more change by looking for more things to improve, based upon previous successes, analysing every win by asking what went right and what needs improvement? Setting goals to continue building momentum, adopting a continuous improvement philosophy (kaizen) and keeping ideas fresh by bringing in new change agents and leaders to form a coalition. 
Step 8: Anchor new approaches in the culture to ensure that they are part of the core of the organisation, hence the values behind the vision show in day-to-day work through continuous improvement in every aspect of the organisation. For Kotter (2012), leaders, new and existing staff, have to continually support the change by talking about progress and success stories, incorporating change ideals in hiring and training new staff and acknowledging key members' contributions.

\section{INTEGER and change management theory}

During the FP7 INTEGER (2015) project, institutional partners in Ireland, France and Lithuania used their project resources to create a sense of urgency and to reinforce the high-level policy shift that each institution had to activate, drawing upon key actors, as change agents, in their individual implementing institutions: Trinity College, Dublin (TCD) Ireland; the Mission des Femmes in (CNRS), France and the Siaulai University, Lithuania. A key lesson learned from INTEGER was that senior management need to be committed to the changes and to demonstrate this visibly. The underlying vision in each INTEGER institution was enshrined in the acronym INstituting Transformation for Effecting Gender Equality in Research and the design of a specific INTEGER logo that reflected and disseminated this. Alongside the use of the acronym and logo and in order to make the necessary gender equality actions more accessible and comprehensible Trinity College, Dublin (TCD) designed the visual metaphor of the INTEGER wheel, as the roadmap action plan framework to be followed. The INTEGER wheel was then adapted for use in the other INTEGER partner institutions. (It was further adapted and refined in 2015 for use as the SAGE wheel.)

INTEGER introduced important and timely communications in the form of: cascading, networking, conferences, exchanges of experience, site visits, presentations of survey findings and recommendations for action and, most importantly, adoption of the INTEGER project's objectives by the university's governing bodies. The TCD project partners produced a video Driving Excellence through Gender Equality featuring the provost and vice provost pledging their support for gender equality and diversity in the university. This served a number of functions in addition to communicating change, it represented a short-term win and demonstrated the buy-in of senior management. To further anchor and reinforce the university's strategic commitment to gender equality, TCD ensured that the Athena SWAN initiative was incorporated into the university's strategic plan 2014-19. Further information on Athena SWAN is provided in Chapter 12.

Initially, change within TCD was driven by internal forces underpinned by WiSER's mantra of: recruiting, retaining and progressing women students and staff in STEM. To change attitudes and behaviours that impede progress towards gender equality, namely perceptual, emotional, cultural and cognitive blocks, external expert speakers were invited to lead unconscious bias awareness sessions in all three INTEGER institutions. TCD adopted a cascade process, working from the top down with key segments of the university, including the promotion 
committees. The INTEGER experiences and outputs were harnessed and built upon still further to inform the next phase of transformation and testing of the SAGE (Systemic Action for Gender Equality) model of actions in GEPs.

\section{The SAGE model for institutional change}

The SAGE model for institutional change (Figure 10.3) built upon the theories of change management experts such as Kanter (1989) and Kotter (2012) along with the experiences of the 2011-2015 INTEGER project, to identify the key components for effective management of change towards gender equality in academia.

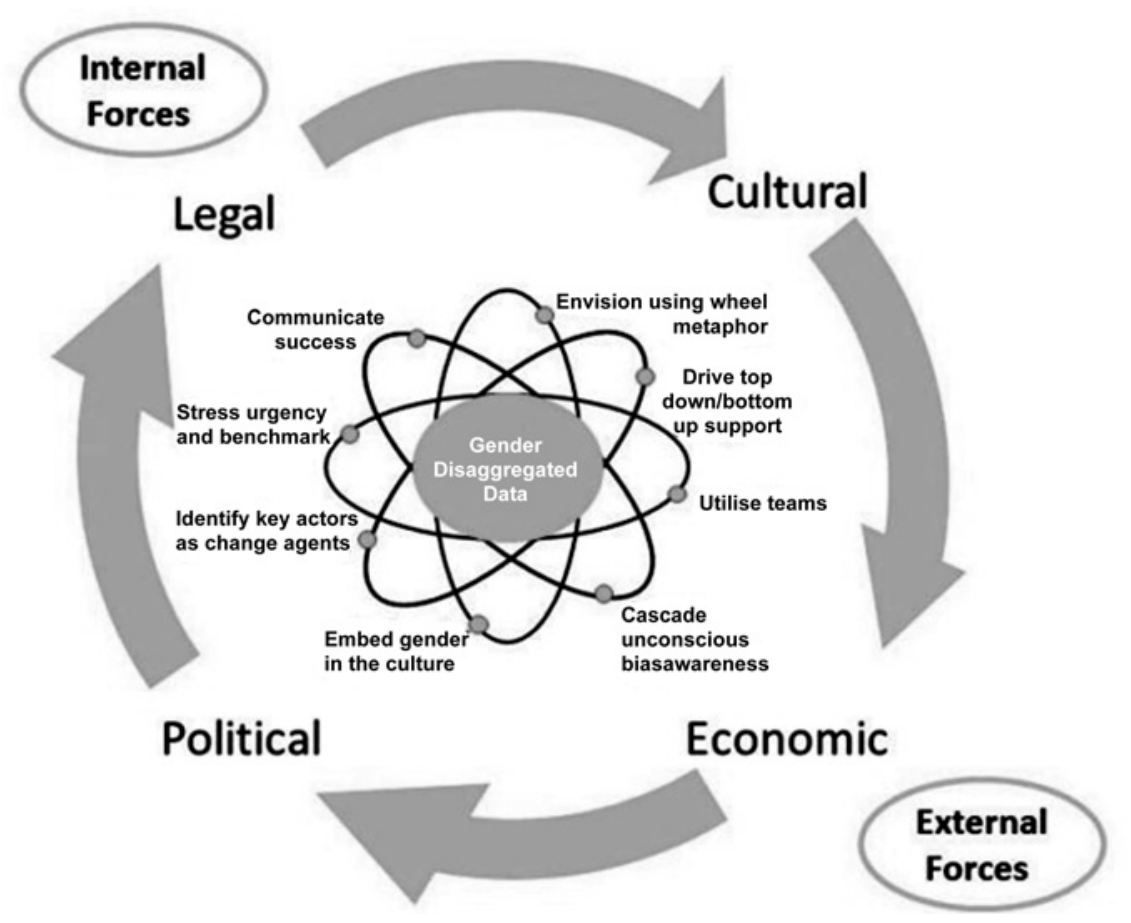

Figure 10.3 SAGE model for institutional change

\section{Gender disaggregated data}

The requirement to monitor gender equality using detailed and accurate data, disaggregated by gender, is at the core of the change management process (Danowitz 2008). Robust data collection provides both the evidence that gender inequalities exist; how pronounced they are; the benchmarking required to measure progress; and to provide comparisons with other institutions. This transparent data collection allows institutions to assess 'whether time and resources are being expended effectively or just wasted' (Kang and Kaplan 2019, 583). 
During the INTEGER project, data were deemed essential to defining the problem and identifying opportunities. Each INTEGER institution embarked upon a thorough data collection exercise, through focus groups and surveys, and a review of policies, procedures and practices to identify barriers to gender equality and draw up the actions necessary to address these. Given that the experience garnered during the INTEGER project illustrated how data are essential to outlining the problem and detecting areas for action relating to gender, the collection, analysis and presentation of gender-disaggregated data were placed at the core of the $S A G E$ wheel and the SAGE model for institutional change. Data collection underpins all the actions that form the Gender Equality Plan (GEP) for the unit (centre, department, school, faculty) and institution.

Each SAGE institution embarked on a thorough data collection exercise through focus groups and surveys and conducted a review of policies, procedures and practices, in order to identify barriers to gender equality and to draw up the actions necessary to address these. This involved collecting gender-based:

- quantitative data on student numbers at all levels; staff numbers of all grades and categories; a gender profile of decision-makers; committee representation; take-up of leave arrangements;

- qualitative data on attitudes, experiences of promotion processes, career aspirations, work-based culture.

Quantitative data help to address the 'what?' question while only qualitative data can address the 'why?' and 'how?' questions, critical to addressing gender inequalities. Hence the most relevant, targeted actions need to be based on a combination of the two types of data. What follows are the responses collected in a SAGE project focus group with partners, conducted via skype. These responses have been anonymised to protect identity and confidentiality. The SAGE partners described this process as a vital starting point:

'You can see where the institution is and where it wants to go-that's a huge thing'.

'Collecting gender-disaggregated data, very basic but very important'.

The practicalities of collecting gender disaggregated data relied on a range of departments, including human resources, student offices and research and evaluation officers. Some of the SAGE partners experienced problems in collecting this gender-disaggregated data. Despite declared support from senior management, some staff were reluctant to allocate the time and resources necessary for this task.

Part of the gender disaggregated data collection involved conducting a survey on the gender culture of the institution. In some cases, this data collection tool proved difficult to administer. For example, if an institution was small, the issue of the anonymity of responses arose:

'There was a problem related to anonymity, due to the small number of staff'. 
Collecting data also allows for benchmarking to compare trends and patterns, policies and processes with other similar organisations. This helps identify existing inequalities and future changes which can then be monitored. Different institutions face different priorities and challenges which is the reason that Gender Equality Plans need to be appropriate and realistic for the specific environment of the individual institution. This reinforces Danowitz's view that 'gender equality initiatives must be tailored to the particular needs of the university and must be adapted to its mission and culture' $(2008,97)$.

\section{Stress urgency and benchmark}

Moss Kanter's concept of 'departures from tradition' and Kotter's emphasis on establishing a sense of urgency can be seen as the initial driving forces for change. Sporn (1999) reiterates this by highlighting how universities adapt their structures in response to environmental forces. 'A key element ... was the notion of crisis or opportunity that created the impetus for change to both the institutional and societal environment' (Sporn 2019, 38). Danowitz (2008) emphasised that gender equality measures must also be linked to major programmes such as the Athena SWAN awards in the UK and Ireland. SAGE partners reported that involvement in the project itself also provided momentum for action:

'The project gave us a more orderly framework for things that were already going on at the university. The external context is very 'anti gender' in [country], but internally we had already been doing some things'.

\section{Identify key actors as change agents}

Kanter (1989) stressed that change agents need to be the right people, in the right place at the right time, to support innovation, encourage building of coalitions and teams to design and implement visions. In doing so, they must influence and persuade those who will be instrumental in bringing about change. The INTEGER experience illustrated that key actors as change agents in the partner institutions played a major role in driving the project forward. Similarly, SAGE partners found that there were key individuals who helped to drive the change forward:

'Once you convince the rector your life gets a lot easier. The head of human resources was also a key person for us'.

\section{Drive top-down and bottom-up support}

Kotter refers to the 'guiding coalition' as vital for success, crucial in seeking buy-in and commitment from senior managers (presidents, deans and so on) to embark upon, or endorse, the necessary actions. Equally, it is essential to activate grass-roots support from those who may serve on teams and/or become change 
agents. As O'Connor's account of change within higher education institutions recognises that:

those in senior positions were much more open to arguments concerning the development of the university, whereas those below this level were much more driven by personal agendas.

Recognising that top-strategic-level buy-in was critical to ensuring changes at all levels, each INTEGER institution invited their president, director, rector or provost to attend and present at INTEGER 'Exchange of experience' project meetings to which distinguished guest speakers from the US and EU were invited to present the case for institutional transformation for gender equality and embedding gender into the university's culture.

The support of senior management, particularly from the director or rector, was crucial to SAGE implementation in all institutions, in addition to human resources staff, given their involvement in the critical areas of recruitment, career development and staff training. The SAGE partners generally found that if the senior management supported the gender equality initiatives this provided essential momentum:

'When we wanted to get something done or we had resistance (which we did) we would communicate that to the director and things would get sorted out'. 'The changes came from a top down direction. We tried to disseminate from some high authority places. ... It is too hard to change from the ground up, as that would mean changing the mind of a lot of people. So we went for the short cut through using the high-up positions, to communicate and then instruct'.

This suggests that, at least initially, top-down support serves as a more effective driver than bottom-up support towards gender equality implementation.

\section{Utilise teams}

Setting up a Gender Equality Plan implementation team is important to achieve: a shared ownership of the vision; broader engagement; and diverse perspectives (gender, age, discipline, seniority, academic, professional and so on). Team members need to be invited to join on the basis of sharing the team's workload towards achieving actions and the organisational gender equality vision. Teams act as the structural drivers of the institutional change process in academia, with members appointed to implement the GEPs and monitor gender equality. Team members have to act as advocates for gender equality initiatives; work to overcome resistance encountered; and pave the way for lasting transformation.

All INTEGER partners formed teams to effect changes, though membership changed, as members exited and were replaced by new entrants. Team membership required a commitment to the process in their role as prime movers. One 
of the 'lessons learned' from INTEGER was that implementation teams should include mixed genders, junior and senior staff, academic and non-academic staff (management, administrative and technical):

'The secret is to involve many people so that they are aware what is the purpose [of the plan] and who the coordinator talks to for this or that'.

The value of creating a diverse GEP implementation team was reinforced by the SAGE experiences, where this approach had not been taken. One partner explained how, although they attempted to work as a team, the small size of the institution made it challenging:

'We found it easier to go individually from one person to another to get the cooperation we needed'.

The same team member reflected that with hindsight, they would have tried a different approach, to find more people to help with the project. Another team member from a different institution also agreed with this:

'We didn't form an institutional-wide team ... [with hindsight] I would create a team all over the university, take more of a ground-up approach'.

\section{Cascade unconscious bias awareness}

Rolling out a programme of unconscious bias awareness training to staff connects with Kotter's emphasis on communicating the change vision and the justification for positive interventions to address bias. Providing training in unconscious bias awareness and its impact is an important first step in communicating the relevance of gender inequality in higher education institutions. It is also important that it is part of a much broader commitment:

to create systems and environments in which bias and stereotyping are either less likely to become initiated, or are prevented from resulting in discrimination even when they are active.

(Kang and Kaplan 2019, 580)

Experience from the INTEGER project demonstrated that unconscious bias and gender awareness sessions need to be scheduled for all levels, ideally starting at the top (senior management) and cascading to lower levels. Top-down attendance is symbolic and sends a signal to other levels to ensure attendance. As Kang and Kaplan point out:

if everyone else in an organisation appears to value diversity, we are more likely to act like we value diversity ourselves ... the most important source of such normative change is that of a group's leaders. 
To change attitudes and behaviours that impede progress towards gender equality, namely, perceptual, emotional, cultural and cognitive blocks, external experts and speakers were invited to lead unconscious bias awareness sessions in all three INTEGER institutions. TCD adopted a cascade process, working from the top-down with key segments of the university including the promotions committee.

All SAGE institutions ran unconscious bias training workshops targeted at different groups within their organisations. Some found that gathering the qualitative data on the existing gender culture through focus groups also served a method for raising awareness of gender equality issues more generally and contributed to overcoming some ideological issues:

'The common prejudice or stereotype is that gender is related to women only, so it's not men's problem'.

Alongside the unconscious bias awareness training, institutions also ran seminars presenting the gender disaggregated data and explaining the impact of inequality. These training events also seemed to be a site where resistance or scepticism about the change process and its value to the institution were revealed:

'We had to use the literature that proves that gender equality gets the whole institution to a higher and more efficient level'.

The experiences of the INTEGER project also provided information on resistance to change, with the lesson learned that some resistance is inevitable. Emphasising the evidence base for good practice, and the benefits to the broad institutional community, helps to maximise support. As is clear from the experiences of the SAGE partner institutions, any efforts to generate significant change are likely to be met with resistance from some quarters and resistance is an inevitable part of the change process. Strategies for managing resistance to change have been dealt with at length in the FESTA project, which produced the Handbook on Resistance to Gender Equality in Academia (2016).

\section{Envision using SAGE wheel metaphor}

For Kotter $(2012,71)$, 'Vision refers to a picture of the future with some implicit or explicit commentary on why people should strive to create that future'. Hence, developing a clear vision and the strategies to achieve that vision play a crucial role in the change process. As previously noted, the underlying vision in each INTEGER institution was enshrined in the acronym/logo and through the use of the INTEGER Wheel, which acted as a roadmap for the GEP.

In the case of the SAGE project, the SAGE wheel model for Gender Equality Plans was used to illustrate the range of actions that can be employed to drive gender equality in higher education institutions. Integrative actions to implement 
change, utilising training and communication are required for people to learn how to use or incorporate the new actions. Kanter recommended devices such as cascading, networks, conferencing, road shows and informal visits to relay information from the converted to prospective and necessary participants. These mechanisms create momentum and critical mass in convincing people to adopt new practices leading to appropriate and timely gender actions.

SAGE partners reported finding the SAGE wheel a useful tool in identifying potential actions for the development of each individual institution's GEP:

'The SAGE model [was] extremely useful as a guideline'.

Despite having the resource of the SAGE model to draw on, some of the SAGE partners reported finding it hard to communicate the purpose of the project to the wider institutional audience.

\section{Embed gender in the culture}

Kotter (2012) advocated anchoring new approaches in the culture and consolidating gains to produce more change, by hiring or promoting people who can implement the change and reinvigorating the process with new projects. For Kang and Kaplan $(2019,581)$, 'Gender inclusive workplace cultures are those that create a positive social climate for people of all gender identities'. The institutional culture needs to be, or become, in tune with the vision of gender equality, otherwise nothing will change. Hence the need to make gender more visible, including LGBTQ+ issues, through diverse speakers/films/events, drawing attention to gender-related issues, such as harassment. Measures to address language, for example in recruitment adverts and promotion calls, need to be gender sensitised. This is a critical stage for building awareness of an inclusive culture that values diversity. In 2019, the Trinity Centre for Gender Equality and Leadership adopted the slogan: 'gender matters-whatever your gender' as part of its contribution to the TCD equality trail. The language and message should, wherever possible, be included in all the institution's plans, reviews and mission statements.

In TCD, it became clear that in order to anchor and institutionalise the gains from INTEGER, and build upon them, required an external stimulus. This was provided by the establishment of an Athena SWAN national committee which led, in turn, to the extension of the Athena SWAN awards to all Irish universities and institutes for higher education. SAGE institutions also produced documents and tools to support the implementation of their GEPs. For example, a guide for those involved in recruitment, and a manual of good practice was produced. Other focus group members referred to career progression, gender in the curriculum and gender in research.

'We have a lot of students who have babies, so we refurbished a room for them to use for baby changing; it is highly utilised'. 


\section{Communicate success}

Kotter (2012) advocates generating short-term wins and anchoring new approaches in the culture by highlighting the connections between new behaviours and organisational success. The communication of 'small wins' (Correll 2017,744 ) is an important part of the incremental route to gender equality since this 'approach can produce important changes in the short run, such as reducing the biases women experience during workplace evaluations, and this change can inspire larger change' (Correll 2017, 745).

INTEGER introduced an important and timely action vehicle to communicate successes through: cascading, networking, conferences and exchanges of experience, site visits, presentations of survey findings/recommendations for action and, most importantly, their adoption by governing bodies (council and board). It also sponsored a media training event run in 2014 to assist INTEGER partners in communicating their message. A similar event was run for SAGE partners in 2018. This enabled SAGE partner institutions to communicate the successful implementation of their GEPs though their institutional social media accounts, communication events, as well as networking with colleagues both internally and externally:

'Once we started, the institution realised it could be useful'.

Evidence emerged of a growing awareness of the SAGE project through, in a number of cases, SAGE teams being proactively approached, internally and externally, for their inputs. The crucial role that communication plays in facilitating a wider support for gender equality measures goes in tandem with the need for SAGE team members to convince colleagues that, not only would improvements in gender equality benefit the institution, but that gender equality was not a threat to academic merit:

'A lot of them saw the gender equality agenda as taking something out of a merit-based university. They were very cautious and some expressed a fear of a merit-based system being attacked from the gender equality side. We needed to make an efficient way to explain how the two can co-exist . . . merit and gender equality are not [mutually] exclusive'.

Accepting that generating significant change is likely to be met with opposition from some quarters is important in overcoming that resistance. Creating and learning new systems, processes and structures takes time, as people build up their competence through integrating knowledge and applying it to their actions. As Hultman concludes:

overcoming resistance is essentially a process of impacting people's facts, beliefs, feelings, values, and behaviour. Some methods for dealing with facts 
and beliefs are to verify facts, clarify beliefs, challenge unviable beliefs, and suggest more viable beliefs.

In the context of the SAGE project, team members worked hard to challenge the belief that gender inequality undermines meritocracy.

\section{Conclusion}

This chapter has outlined the role played by change management theories and the lessons learned from previous gender equality initiatives in developing the SAGE model for institutional change. Alongside this, the chapter has presented the reflections of the SAGE team members on putting the model into practice, as they worked towards implementing institutional GEPs. The SAGE process is perceived as a starting point for gender equality by the SAGE team members, who are realistic about how much change can be achieved during a three-year project, particularly when academic careers in some countries can take many years to develop:

'We have only two female professors and a low representation of women. You're not going to solve that in three years'.

'A three-year project is too short a time to implement such change. The time for career change is very long, so it's hard to see change in three years. Not just for accepting the idea that gender is something we should invest in, but career change is very lengthy process'.

However, the value of the SAGE model is best summed up by the team member who said:

'The SAGE model gave us directions where to start - collecting data - and four areas of action. You need someone to guide you . . . it provides a structure, but you need guidance too'.

\section{References}

Benschop, Y. and Verloo, M. (2011) Gender, change, organizational change and gender equality strategies, in E. Jeanes, D. Knights and P. Martin (eds), Handbook of gender, work and organization, John Wiley \& Sons, Chichester.

Clark, B. (1998) Creating entrepreneurial universities: Organizational pathways of transformation. Issues in higher education. Elsevier Science, New York.

Clark, B. (2004) Sustaining change in universities: Continuities in case studies and concepts, McGraw-Hill Education, London.

Correll, S. (2017) Reducing gender biases in modern workplaces: A small wins approach to organisational change, Gender and Society, 31 (6), 725-750, doi:10.1177/08912432 17738518 
Danowitz, M. (2008) Gender equality as organizational change: Frames, challenges, and strategies in the EU and US, in S. Grenz, B. Kortendiek, M. Kriszio and A. Löther (eds), Gender equality programmes in higher education: International perspectives, VS Verlag, Wiesbaden, 87-100.

FESTA (2016) Handbook on resistance to gender equality in academia, Female Empowerment in Science and Technology Academia: FESTA. Available at: www.festa-europa.eu/ public/handbook-resistance-gender-equality-academia

HEA (2016) HEA national review of gender equality in Irish higher education institutions, Higher Education Authority, Dublin.

Hultman, K. (2003) Managing resistance to change, Encyclopedia of Information Systems, 3, 693-705.

INTEGER (2015) Institutional transformation for effecting gender equality in research. Available at: www.integer-tools-for-action.eu/en/resource/lessons-learned

Kang, S. and Kaplan, S. (2019) Working towards gender diversity and inclusion in medicine: Myths and solutions, Lancet, 393, 579-586, doi.org/10.1016/S0140-6736(18)33138-6

Kanter, R. M. (1977) Men and women of the corporation, Basic Books, New York.

Kanter, R. M. (1989) The change masters corporate: Entrepreneurs at work, Allen and Unwin, London.

Kotter, J. (2012) Leading change, Harvard Business Review Press, Cambridge, MA.

O'Connor, P. (2019) An autoethnographic account of a pragmatic inclusionary strategy and tactics as a form of feminist activism, Equality, Diversity and Inclusion: An International Journal, 38 (8), 825-840, doi.org/10.1108/EDI-12-2018-0227

O’Connor, P., Martin, P., Carvalho, T., O’Hagan, C., Veronesi, L., Mich, O., Saglamer, G., Tan, M. and Caglayan, H. (2019) Leadership practices by senior position holders in higher educational research institutes: Stealth power in action, Leadership, 15 (6), 722-743, doi.org/10.1177/1742715019853200

Sporn, B. (1999) Adaptive university structures: An analysis of adaptation to socioeconomic environments of US and European universities, Higher education policy series No. 54, Taylor and Francis, Philadelphia.

Sporn, B. (2019) Adaptive university structures - from theory to practice and back, in P. Teixeira, A. Veiga, M. Machado, P. da Rosa and A. Magalhães (eds), Under pressure: Higher education institutions coping with multiple challenges, Koninklijke Brill NV, Leiden. 